\title{
Rapid and simple detection of endospore counts in probiotic Bacillus cultures using dipicolinic acid (DPA) as a marker
}

\author{
Xiao-Sheng Liang, Chun Liu, Zhu Long and Xiao-Hua Guo*
}

\begin{abstract}
Spore counting in probiotic Bacillus cultures using dipicolinic acid (DPA) as a marker was studied for developing a rapid and simple detection method. The newly developed method is based on the fluorescence enhancement by a new chelating agent, which forms a complex with $\mathrm{EuCl}_{3}$ and DPA. The results showed that 1,2-cyclohexanediamine$N, N, N^{\prime} N^{\prime}$-tetraacetic acid (CyDTA) greatly enhanced the fluorescence intensity in all selected chelating agents. The optimal composition of the fluorescence complex DPA-Eu-CyDTA had a detection limit of $0.3 \mathrm{nM}$ of DPA. Metal ions in high concentrations, including $\mathrm{Cu}^{2+}, \mathrm{Fe}^{2+}, \mathrm{Fe}^{3+}, \mathrm{Al}^{3+}$, and $\mathrm{Zn}^{2+}$, might lower the detection sensitivity, which could be eliminated by diluting the sample with the metal ions below $10 \mu \mathrm{M}$. The maximum release of DPA was achieved by heating treatments at $121^{\circ} \mathrm{C}$ for at least $10 \mathrm{~min}$ for two types of Bacillus endospores. The spore concentrations and corresponding released DPA fluorescence intensities were linearly associated (coefficient $R^{2}=0.9993$ and 0.9995 for Bacillus subtilis MA139 and Bacillus licheniformis BL20386, respectively). The detection limit for both strains reached approximately 6800 spores $/ \mathrm{mL}$. The verification results showed that the DPA fluorimetry assay developed in the present study was fully consistent with the plate-counting assay. The study shows that the fluorescence complex DPA-Eu-CyDTA can be reliably used for the detection of endospores in Bacillus fermentation for the production of probiotics.
\end{abstract}

Keywords: Bacillus, Probiotics, Endospore detection, Dipicolinic acid, Fluorescence

\section{Introduction}

Bacillus species have been extensively studied for use as probiotics during the past 20 years both for scientific exploration and commercial development (Hong et al. 2005; Cutting 2011). Spore probiotics are widely used in humans for medical purposes as dietary supplements for preventing diarrhea and intestinal disorders. The strain of Bacillus licheniformis BL20386 used in this study is the main ingredient in probiotic Zhengchangsheng ${ }^{\circledR}$ capsule, a useful therapeutic agent for the treatment of diarrhea (Heo et al. 2014). In the field of animal nutrition, spore probiotics enhance the growth performance and diseaseresistance of poultry, swine, and shrimps (Cutting 2011).

\footnotetext{
*Correspondence: guo_xh@hotmail.com

Provincial Key Laboratory for Protection and Application of Special Plants in Wuling Area of China, College of Life Science, South-Central University for Nationalities, No. 182, Minyuan Road, Hongshan District, Wuhan 430074, Hubei, China
}

The B. subtilis MA139 strain, also used in this study, has been applied as feed probiotics, benefiting piglets' growth and modifying intestinal microflora (Guo et al. 2006). Except for non-spore Lactobacillus probiotics, endospores are extremely heat stable and acid resistant, which enables long-time storage without loss of viability during storage at room temperature and survival when passing through the gastric barrier.

In the production of probiotics from spore formers of Bacillus, the growth and accumulation of spores are carefully monitored to ensure a high fermentation yield (Zhao et al. 2008; Khardziani et al. 2017). To lower the cost of high spore production, maximum spore yields are often optimized before scale-up fermentation (Pryor et al. 2007; Chen et al. 2010). In any situation, spore counts must be accurately quantified to control the fermentation. The routine method for spore quantification often relies on plate-counting assays, which generally are 
a time-consuming and quite tedious method (Hazan et al. 2012). In recent years, numerous researchers have studied the development of rapid methods for spore detection that can be partial alternatives to routinely monitoring spores (Hindle and Hall 1999; He et al. 2003; Fichtel et al. 2007; Postollec et al. 2010; Bai et al. 2014; Hebert et al. 2017). Many analytical techniques have been based on the detection of dipicolinic acid (DPA) in endospores as a unique constituent, including high-performance liquid chromatography (HPLC), photofluorescence, and capillary zone electrophoresis (Hindle and Hall 1999; Pellegrino et al. 2002; Fichtel et al. 2007; Ai et al. 2009). With its tridentate ligand structures, DPA can specifically form a complex with terbium (Tb), one of the lanthanides, and exhibit enhanced luminescence when irradiated by UV light. This method has a limit of detection (LOD) of $2 \mathrm{nM}$ of DPA (Hindle and Hall 1999). Combined with the technique for DPA released from endospores, the reported LOD for the Tb-DPA luminescence method reached $10^{3}-10^{4}$ B. subtilis spores per $\mathrm{mL}$ (Hindle and Hall 1999; Pellegrino et al. 2002). However, the LOD for DPA based on Tb-DPA fluorescence often depends on the concentration of reagents (Hindle and Hall 1999), which might cause trouble in the simultaneous detection of different samples with various DPA contents. Meanwhile, excess $\mathrm{Tb}$ in the complex produces high background fluorescence and leads to a lower LOD in the detection (Hindle and Hall 1999; Ai et al. 2009).

In the present study, another DPA-Eu-based luminescence method was explored for simple detection and a lower LOD for DPA. Two strains, B. subtilis MA139 and $B$. licheniformis BL20386, were used as models to assess the endospore counts based on a DPA fluorimetry assay, which was studied for monitoring and optimizing spore production in probiotics commercialization.

\section{Materials and methods \\ DPA fluorimetry assay}

DPA standards with a purity of $99 \%$ were purchased from the Aladdin Company (Cat. No. P109609; Shanghai, China). A standard stock solution of DPA with a $10 \mathrm{mM}$ concentration was prepared by dissolving $161.72 \mathrm{mg}$ of DPA in $100 \mathrm{~mL}$ of Tris-HCl buffer $(50 \mathrm{mM}, \mathrm{pH} 8.0$; the same below). The stock solution was stored at $4{ }^{\circ} \mathrm{C}$ and diluted by Tris- $\mathrm{HCl}$ buffer to produce working solutions at different concentrations before use. Two common lanthanide ions, $\mathrm{EuCl}_{3}$ and $\mathrm{TbCl}_{3}$ (Alfa Aesar, 99.9\% purity), were selected for possible fluorescence enhancement when they chelated with DPA in the presence of chelating agents. Two $\mathrm{mM}$ of $\mathrm{EuCl}_{3}$ and $\mathrm{TbCl}_{3}$ were prepared in Tris- $\mathrm{HCl}$ buffer. As structural analogues, four chelating agents were screened for fluorescence enhancement, including ethylenediaminetetraacetic acid (EDTA), 1,2-cyclohexanediamine- $\mathrm{N}, \mathrm{N}, \mathrm{N}^{\prime} \mathrm{N}^{\prime}$-tetraacetic acid (CyDTA), 2-hydroxyethylethylenediamine triacetic acid (HEDTA), and ethylene glycol tetraacetic acid (EGTA). The chelating agents were prepared in Tris- $\mathrm{HCl}$ buffer in $2 \mathrm{mM}$ concentrations. A lanthanide solution was first mixed with an equal volume of a chelating agent solution, and then a $0.4 \mathrm{~mL}$ DPA sample was added to the premixed, $3.6 \mathrm{~mL}$ lanthanide-chelating agent solution to produce the fluorescence complex in a final volume of $4 \mathrm{~mL}$. The fluorescence intensity was detected by a Hitachi F-7000 spectrofluorophotometer (Hitachi Ltd., Tokyo, Japan). The pre-set parameters were a scanning speed of $3000 \mathrm{~nm} / \mathrm{min}$, a $10 \mathrm{~nm}$ slit, $700 \mathrm{~V}$ of photo-multiplier tube (PMT) voltage for Eu-based complexes and $400 \mathrm{~V}$ for Tb-based complexes, and a responding time of $0.08 \mathrm{~s}$. Each time, the fluorescence complexes were freshly prepared and then detected immediately. First, the fluorescence complexes with different chelating agents were scanned from 500 to $700 \mathrm{~nm}$ at a $270 \mathrm{~nm}$ excitation wavelength. The $1.8 \mathrm{mM}$ lanthanide solutions (equal to the same concentration of lanthanides in the fluorescence complexes) were also scanned to detect possible fluorescence intensity. As a control, a mixture of $0.4 \mathrm{~mL}$ DPA sample, $1.8 \mathrm{~mL}$ lanthanide solution, and $1.8 \mathrm{~mL}$ of Tris$\mathrm{HCl}$ was also scanned for a fluorescence peak. Tris- $\mathrm{HCl}$ buffer was used as a blank control. The chelating agent that produced the highest fluorescence peak was selected for DPA detection. The fluorescence complex with the selected chelating agent was further scanned to determine the excitation and emission wavelengths based on the DPA standard in a used concentration.

The linear correlation of the fluorescence intensity coupled with the DPA concentration was tested based on three parallel experiments. In order to improve the accuracy of measurements, the DPA samples were diluted to adjust the light output in arbitrary units on a scale from 0 to 1000 arbitrary units (AU) based on earlier reports (Hindle and Hall 1999; Fichtel et al. 2007).

\section{Metal ions interference}

The effect of metal salts on the fluorescence intensity was studied for possible interference. Ten metal ions $\left(\mathrm{NaCl}, \mathrm{CaCl}_{2}, \mathrm{KCl}, \mathrm{MgSO}_{4}, \mathrm{MnSO}_{4}, \mathrm{CuSO}_{4}, \mathrm{FeSO}_{4}\right.$, $\mathrm{FeCl}_{3}, \mathrm{AlCl}_{3}$, and $\mathrm{ZnSO}_{4}$ ) were dissolved in a $4 \mu \mathrm{M}$ DPA solution to reach the concentrations of $10,1,0.1$, and $0 \mathrm{mM}$, respectively. Then, a $0.4 \mathrm{~mL}$ DPA solution containing a metal ion was mixed with $2 \mathrm{~mL}$ of $\mathrm{EuCl}_{3}$ and $2 \mathrm{~mL}$ of CyDTA to produce fluorescence complexes. The fluorescence intensity of the mixtures containing different ions was then measured. The fluorescence intensity was compared with the control based on three replications. 


\section{Bacterial strain and culture media}

Two representative probiotic Bacillus strains, B. subtilis MA139 and B. licheniformis BL20386, were used in the study. B. subtilis MA139 was deposited in the China General Microbiological Culture Collection Center (CGMCC) as CGMCC 7.90. B. licheniformis BL20386 was obtained from the China National Center for Medical Culture Collections (CMCC) as CMCC 63516. The strains were kept at $-80{ }^{\circ} \mathrm{C}$ in $20 \%$ sterile glycerol before use. Luria-Bertani (LB) media (10 g/L tryptone, $5 \mathrm{~g} / \mathrm{L}$ yeast extract, and $10 \mathrm{~g} / \mathrm{L} \mathrm{NaCl} ; \mathrm{pH}$ 7.0) was used for the strains' cultivation and fermentation. The strains were cultured in rotating shaker at $37^{\circ} \mathrm{C}$ and $200 \mathrm{rpm}$.

\section{Preparation of endospores and spore suspensions}

The strains were grown and sporulated in nutrient sporulation medium (NSM) (Vasantha and Freese 1979) for $48-60 \mathrm{~h}$ at $37^{\circ} \mathrm{C}$. The mature spores were harvested after a heat shock of $80^{\circ} \mathrm{C}$ for $15 \mathrm{~min}$. The spores were purified by washing twice and then were resuspended in Tris$\mathrm{HCl}$ buffer with an $\mathrm{OD}_{600 \mathrm{~nm}}$ of about 1.0 (equivalent to $10^{8}$ spores $/ \mathrm{mL}$ ). Each time the spores were freshly prepared for the experiment.

\section{DPA release from endospores}

Freshly prepared spore suspensions were diluted by Tris- $\mathrm{HCl}$ buffer to a concentration of approximately $10^{7}$ colony-forming units per $\mathrm{mL}(\mathrm{CFU} / \mathrm{mL})$. Then, the dilution was treated immediately for the release of DPA from endospores. The two methods used were heat shock and L-alanine-triggered germination (Pellegrino et al. 2002). As for the heating shock, the spore suspensions were autoclaved in screw-cap glass test tubes at $105{ }^{\circ} \mathrm{C}$ for $15 \mathrm{~min}, 115{ }^{\circ} \mathrm{C}$ for $20 \mathrm{~min}, 121^{\circ} \mathrm{C}$ for $10 \mathrm{~min}$, and $121{ }^{\circ} \mathrm{C}$ for $20 \mathrm{~min}$, respectively. After cooling, the supernatants containing the DPA were collected after centrifugation $(2500 \times g$ for $10 \mathrm{~min})$ and tested for fluorescence intensity. In the other method, the freshly prepared spores were centrifuged and suspended in $100 \mathrm{mM} \mathrm{L}$-Ala solution (in Tris- $\mathrm{HCl}$ buffer). Every 1, 2, and $3 \mathrm{~h}$, the suspensions were sampled for the detection of fluorescence intensity. All the experiments were conducted with three replications.

\section{Spore plate-counting assay}

The traditional plate-counting assay was used to determine the spore concentrations (Chen et al. 2010). Spores were first heated at $80{ }^{\circ} \mathrm{C}$ for $15 \mathrm{~min}$ to kill vegetative cells, and then the spore suspensions were serially diluted in physiological saline. The spore dilutions were plated on an LB agar medium, and the colonies were counted after cultivation at $37^{\circ} \mathrm{C}$ for $24 \mathrm{~h}$. The results were expressed as $\mathrm{CFU} / \mathrm{mL}$.

\section{Validation of spore counting}

The spore suspensions of the two strains, each of which had an initial $\mathrm{OD}_{600 \mathrm{~nm}}$ of 1.0, were treated by autoclaving, and then the supernatants were collected by centrifugation $(2500 \times g$ for $10 \mathrm{~min})$. The supernatants were then serially diluted twofold, and the corresponding DPA fluorescence intensity of the two strains was detected. The initial concentrations of the two spore suspensions were separately detected by plate-counting assays. Correspondingly, a linear correlation between spore concentrations $(\mathrm{CFU} / \mathrm{mL})$ and the fluorescence intensity (AU) was built for the two strains.

The verification experiments for the detection of spore concentration in Bacillus fermentation were carried out by sampling the fermented broths in LB media from the two strains at 12,24 , and $36 \mathrm{~h}$. The samples were washed twice and suspended in an equal volume of Tris- $\mathrm{HCl}$ buffer. The spores were then quantified by a fluorimetry assay and a plate-counting assay. The obtained results were expressed as the means of fluorescence intensity or CFU/mL and their standard deviation (SD) based on three replicated experiments. The spore concentrations based on the fluorimetry assay were further converted to $\mathrm{CFU} / \mathrm{mL}$ according to the standard curves of the two strains. The Student's $t$ test in JMP 11.0 (SAS Institute Inc., USA) was used to test the differences between the two assays. A $P$ value less than 0.05 was regarded as a significant difference.

\section{Results}

\section{DPA standard fluorimetry assay}

The supplementation of chelating agents in DPA-Eu complexes greatly enhanced the production of fluorescence signals by EGTA, HEDTA, EDTA, and CyDTA (Fig. 1a). The supplementation of CyDTA showed the best effect in improving the fluorescence intensity compared with the other three chelating agents. No fluorescence signal was detected in the DPA-Eu complex. The $\mathrm{TbCl}_{3}$ showed high background fluorescence, and the supplementation of chelating agents greatly decreased the fluorescence intensity compared with the Tb-DPA complex (Fig. 1b). The $1.8 \mathrm{mM} \mathrm{TbCl}{ }_{3}$ complex produced a fluorescence intensity of $1847 \mathrm{AU}$ at $546 \mathrm{~nm}$. Chelated with $100 \mathrm{nM}$ DPA, the fluorescence intensity of the Tb-DPA complex only increased to $2057 \mathrm{AU}$.

The fluorescence complex DPA-Eu-CyDTA was further studied for specific DPA detection. The peak height was greatly affected by the excitation light, and the highest peak was observed at the excitation wavelength of $270 \mathrm{~nm}$ (Fig. 2a) and emission wavelength of $619 \mathrm{~nm}$ (Fig. 2b). Under the optimal excitation and emission wavelengths, the fluorescence intensity of the complex was proportional to the DPA concentration over a range 

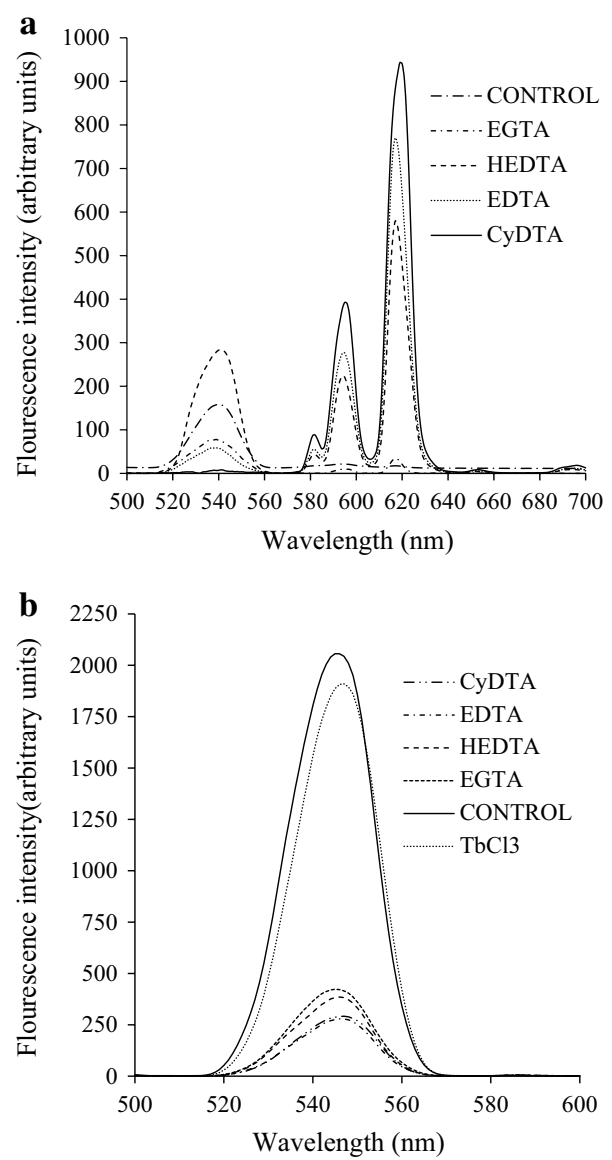

Fig. 1 Effects of different clathrates with $\mathrm{EuCl}_{3}(\mathbf{a})$ and $\mathrm{TbCl}_{3}$ (b) on DPA fluorescence intensity and emission fluorescence spectra. DPA final concentration $400 \mathrm{nM}(\mathbf{a})$, and $100 \mathrm{nM}$ (b)

from 10 to $400 \mathrm{nM}$ with a higher correlation coefficient $\left(R^{2}=0.9979\right.$; Fig. 3). The LOD for DPA was $0.3 \mathrm{nM}$ based on the formula $\mathrm{LOD}=3 \sigma / S$, where $\sigma$ is the $\mathrm{SD}$ of the background fluorescence with ten repetitions and $S$ is the slope of the calibration curve (Rai and Rai 2008).

\section{Effect of metal ions on DPA fluorimetry assay}

In all, ten metal ions were tested in the study. The inclusion of $\mathrm{Na}, \mathrm{Ca}, \mathrm{K}, \mathrm{Mg}$, and $\mathrm{Mn}$ had no effect on the fluorescence intensity of the DPA-Eu-CyDTA complex based on the present study (Fig. 4a). Some other metal ions, including $\mathrm{Cu}^{2+}, \mathrm{Fe}^{2+}, \mathrm{Fe}^{3+}, \mathrm{Al}^{3+}$, and $\mathrm{Zn}^{2+}$, might lower detection sensitivity based on the results in Fig. 4b. However, even with the presence of those ions in the DPA solutions, the concentration of ions could be diluted to be under $10 \mu \mathrm{M}$ to eliminate the interference.

\section{Effect of treating methods on DPA release from endospores} The maximum release of DPA was achieved by heating treatments at $121{ }^{\circ} \mathrm{C}$ for at least $10 \mathrm{~min}$ for both
Bacillus spores (Fig. 5). In this study, B. subtilis MA139 was almost non-responsive to the L-Ala method since only about $25 \%$ of the DPA was released after $3 \mathrm{~h}$ of culturing with L-Ala compared to the heating treatment at $121{ }^{\circ} \mathrm{C}$ for $20 \mathrm{~min}$. The endospores of B. licheniformis BL20386 were more sensitive to L-Ala, and more than $90 \%$ of the DPA was released when treated by $100 \mathrm{mM}$ of $\mathrm{L}$-Ala for $3 \mathrm{~h}$. The full release of DPA was achieved after treatment by $100 \mathrm{mM}$ of L-Ala for $4 \mathrm{~h}$. In terms of the speed and efficiency of DPA release in the two current methods, the heating treatment was preferable for spore quantification, and the treatment at $121{ }^{\circ} \mathrm{C}$ for $10 \mathrm{~min}$ was enough for the full release of DPA for the two strains.

\section{Calibration curves of spore counts based on DPA fluorimetry assay}

Figure 6 shows a good linear correlation between the spore concentrations and corresponding released DPA fluorescence intensity (coefficient $R^{2}=0.9993$ and 0.9995 for B. subtilis MA139 and B. licheniformis BL20386, respectively). The LOD for the both strains reached approximately 6800 spores $/ \mathrm{mL}$.

\section{Verification of spore counting in fermented broth}

The results of spore detection based on DPA fluorimetry and the plate-counting assay are separately presented in Table 1. At each sampling point, no significant differences between the two assays were found for the two Bacillus strains $(P>0.05)$.

\section{Discussion}

Lanthanide ions chelated by DPA show enhanced fluorescence by energy transference. Their unique fluorescent characteristic is often adapted for the determination of DPA from spore-formers in environmental samples (Fichtel et al. 2007). The Tb-based DPA fluorimetry assay has been intensively studied for determining the number of endospores since the DPA-Tb complex shows the advantage of highly enhanced fluorescence and a long fluorescence lifetime (Rosen et al. 1997; Hindle and Hall 1999; Pellegrino et al. 2002). However, in the study by Hindle and Hall (1999), the desired LOD for DPA often relied on excess $\mathrm{Tb}$ compared with DPA, and different concentrations of $\mathrm{Tb}$ could result in different calibration curves corresponding to standard DPA concentrations. Moreover, unchelated $\mathrm{Tb}$ could produce too high background fluorescence (Hindle and Hall 1999), which was in line with the result in Fig. 1b. Therefore, the LOD may depend on desirable concentrations of $\mathrm{TbCl}_{3}$, and the concentrations of reagents are often adjusted before detection (Hindle and Hall 1999). However, in fermentation for spore production, the spore concentration often relies on the culture time and fermentation conditions, 

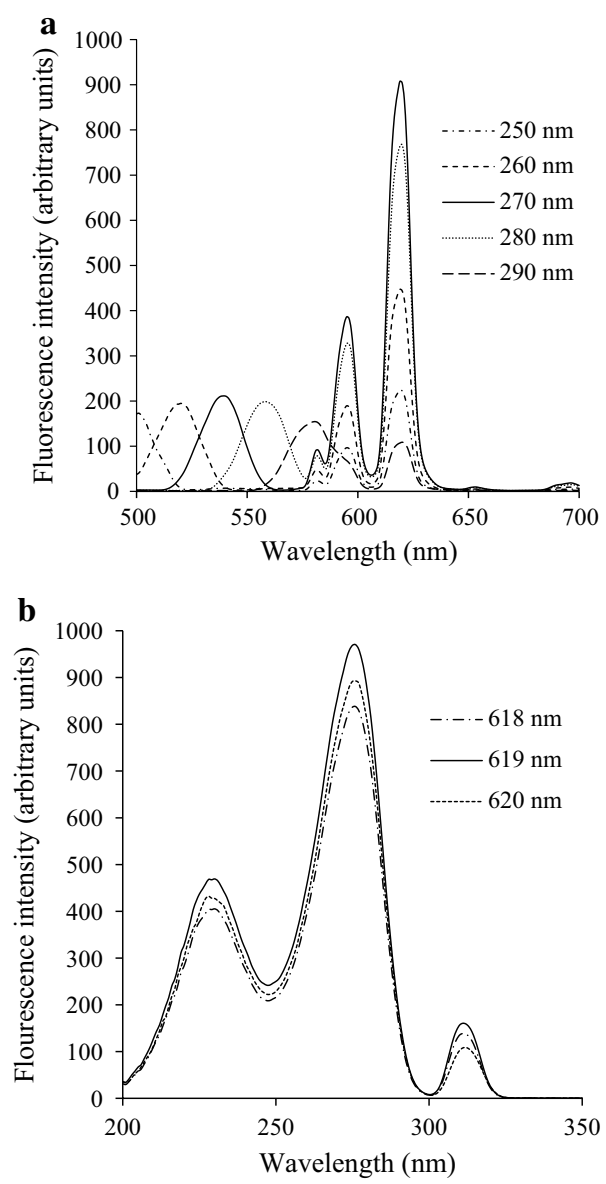

Fig. 2 Emission (a) and excitation (b) fluorescence spectra of DPA-Eu-CyDTA with the DPA final concentration of $400 \mathrm{nM}$

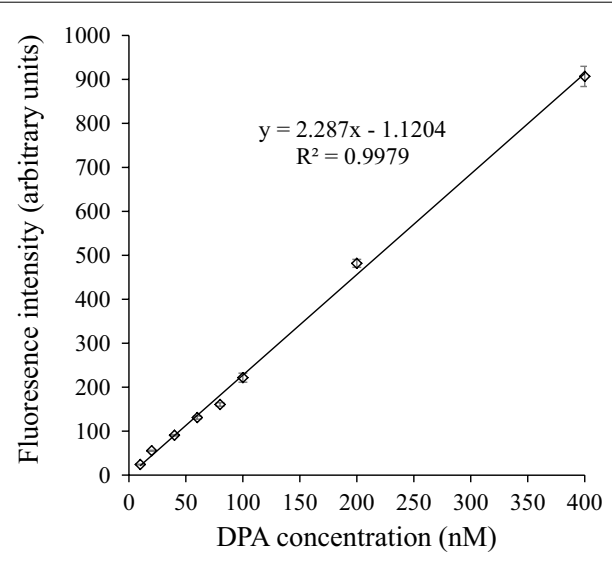

Fig. 3 The standard curve of DPA ranging from 10 to $400 \mathrm{nM}$

and many analytes with different spore concentrations produced in spore optimization designs must be detected simultaneously (Ren et al. 2018). Therefore, the DPA-Tb
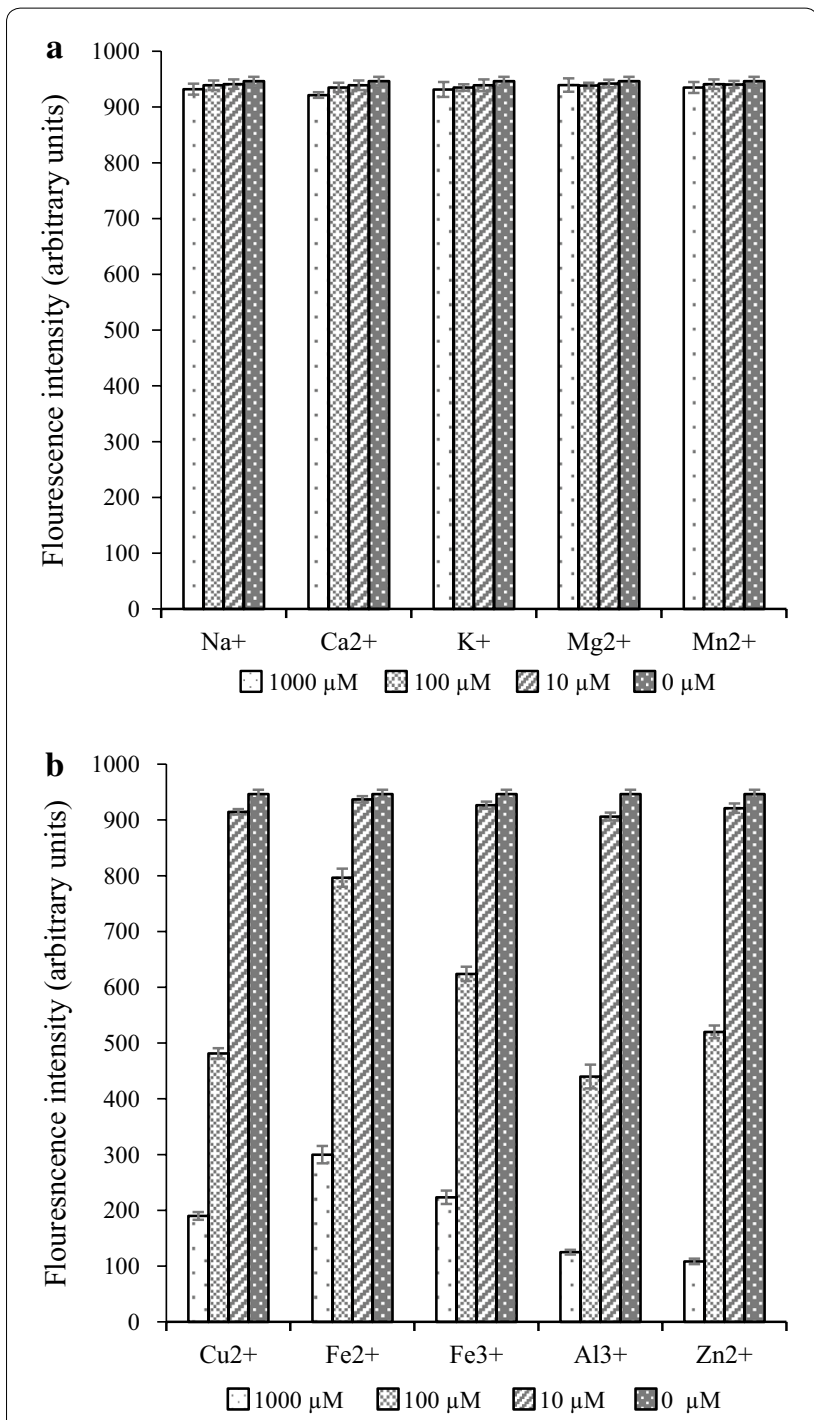

Fig. 4 The effect of different metal ions $\left(\mathbf{a} \mathrm{Na}^{+}, \mathrm{Ca}^{2+}, \mathrm{K}^{+}, \mathrm{Mg}^{2+}, \mathrm{Mn}^{2+}\right.$; b Cu$\left.{ }^{2+}, \mathrm{Fe}^{2+}, \mathrm{Fe}^{3+}, \mathrm{Al}^{3+}, \mathrm{Zn}^{2+}\right)$ at different concentrations (1000, 100, 10 and $0 \mu \mathrm{M}$ ) on the DPA fluorescence intensity

fluorimetry assay cannot be used as a general analytical method for rapid endospore quantification.

Alternatively, Ai et al. (2009) reported a method based on $\mathrm{Eu}$ fluorescence nanoparticle sensor for the possible detection of $B$. anthracis spores. Using Eu-based fluorescence with a red emission offers the advantages of a higher penetrating power and a higher resistance to interferences, which particularly benefit the detection of spores in complex environments. The direct excitation of DPA-Eu complexes produces a weak fluorescent signal, and the background fluorescence can be negligible (Hindle and Hall 1999). Hindle and Hall's results were in accordance with the findings in Fig. 1a. EDTA was often used as a chelating agent for fluorescence enhancement 


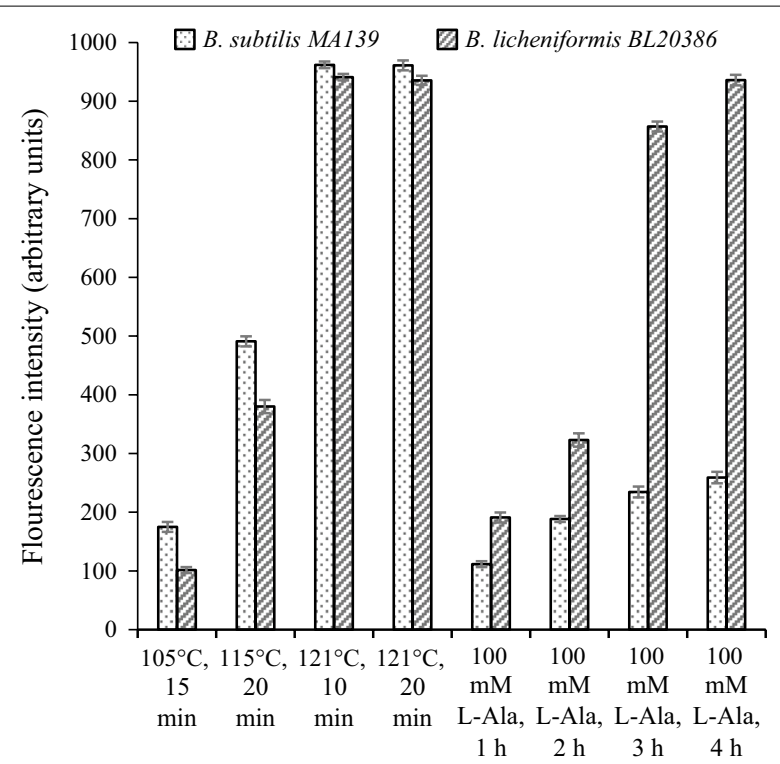

Fig. 5 Effect of different treatments on the release of DPA from endospores of B. subtilis MA139 and B. licheniformis BL20386. The value of fluorescence intensity is based the 30 -fold dilution of the spore suspensions with $\mathrm{OD}_{600 \mathrm{~nm}}=1.0$

(Arnaud and Georges 2003; Ai et al. 2009; Reisfeld et al. 2011). However, in the present study, the complex of DPA-Eu-CyDTA exhibited significantly higher fluorescence intensity compared with that of Eu-EDTA-DPA. This is probably associated with the higher hydrophobicity in the molecular structure of CyDTA than that of EDTA, which lowers the nonradiative quenching of the $\mathrm{Eu}$ emission caused by high-frequency $\mathrm{OH}$ oscillations of water molecules (Ai et al. 2009). This is the first report on fluorescence enhancement by CyDTA in a DPA-Eu complex. The DPA-Eu-CyDTA fluorescence complex provided an excellent linear correlation with DPA concentration with an LOD of $0.3 \mathrm{nM}$ (Fig. 3). The LOD of DPA is close to the value reported by Ai et al. (2009) with $0.2 \mathrm{nM}$. However, the method in this study is simpler, and the fluorescent complex is readily prepared by just mixing the DPA samples, Eu, and CyDTA in the correct proportion. The method is especially suitable for the circumstances of the spore detection in fermentation optimization and quality control and also for the simultaneous detection of endospores in a large number of samples (Ren et al. 2018).

The DPA in bacterial spores exists in the form of a CaDPA complex. Therefore, the release of DPA from endospores could also be caused by the addition of $\mathrm{Ca}^{2+}$ in the solution. Fortunately, the presence of $\mathrm{Ca}^{2+}$ showed no interference, even with a high concentration of $1 \mathrm{mM}$. The interference of other ions on fluorescence detection depends on the ion concentrations. The interference can
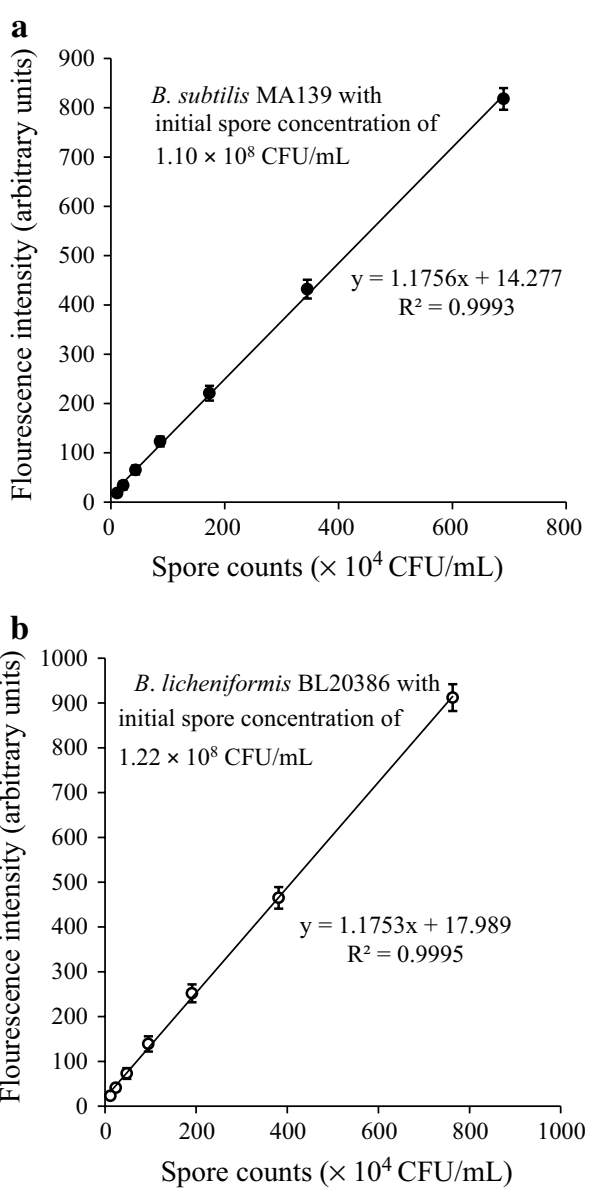

Fig. 6 Calibration curves of spore counts of B. subtilis MA139 (a) and B. licheniformis BL20386 (b) and their fluorescence intensity

be eliminated if the concentration is diluted to less than $10 \mu \mathrm{M}$. In practical samples from spore fermentation, the spore suspensions are often treated by washing and above 100-fold dilutions (Ren et al. 2018). The possible metal ions are then greatly diluted, and their interference is likely to be negligible.

L-Ala has often been the primary germinant to induce spores' germination and the release of DPA into the solution (Setlow 2003). The present results showed that the bacterial endospores of different species had diverse responses to the same germinants (Fig. 5), which was also verified in Vanderberg's report (2000). However, conducting a heating treatment for at least $10 \mathrm{~min}$ led to the full release of DPA for both strains. In the report by Pellegrino, all available DPA was extracted by autoclaving at $121{ }^{\circ} \mathrm{C}$ for $30 \mathrm{~min}$. The difference in treatment time might be associated with the ramp time in different experimental conditions and the specific strains. In consideration of the difference of endospores, a slightly longer autoclave treatment was 
Table 1 The confirmative results on spore detection by DPA fluorimetry assay of B. subtilis MA139 and B. licheniformis BL20386 compared with Plate counting assay

\begin{tabular}{llclll}
\hline Sampling time (h) & \multicolumn{2}{l}{ B. subtilis MA139 concentration (CFU/mL) } & & \multicolumn{2}{l}{ B. licheniformis BL20386 concentration (CFU/mL) } \\
\cline { 2 - 3 } & DPA fluorimetry assay & Plate counting assay & & DPA fluorimetry assay & Plate counting assay \\
\hline 12 & $(4.35 \pm 0.21) \times 10^{4}$ & $(4.05 \pm 0.35) \times 10^{4}$ & & $(5.85 \pm 0.63) \times 10^{4}$ & $(5.55 \pm 0.78) \times 10^{4}$ \\
24 & $(5.65 \pm 0.21) \times 10^{5}$ & $(5.50 \pm 0.36) \times 10^{5}$ & & $(8.30 \pm 0.28) \times 10^{5}$ & $(7.90 \pm 1.27) \times 10^{5}$ \\
36 & $(4.32 \pm 0.11) \times 10^{7}$ & $(3.80 \pm 0.35) \times 10^{7}$ & & $(4.70 \pm 0.14) \times 10^{7}$ & $(4.30 \pm 1.27) \times 10^{7}$ \\
\hline
\end{tabular}

suggested to ensure the full release of DPA (Pellegrino et al. 2002). In terms of the two currently used methods' speed and efficiency of DPA release, the heating treatment appears to preferable for spore quantification. In the present study, the LOD of approximately $6800 \mathrm{CFU} / \mathrm{mL}$ for both strains was close to the level in the earlier reports by Pellegrino $\left(10^{3} \mathrm{CFU} / \mathrm{mL}\right)$ and Alistair $\left(10^{4} \mathrm{CFU} / \mathrm{mL}\right)$. According to the verification experiment shown in Table 1, the results indicated that the spore count detected by the fluorimetry assay was completely consistent with the plate-counting assay.

In conclusion, this study provided a rapid and simple detection method for quantifying Bacillus probiotics endospores based on a new chelating agent to form the fluorescence complex DPA-Eu-CyDTA. The optimal system in the fluorescence complex DPA-EuCyDTA had a detection limit of $0.3 \mathrm{nM}$ of DPA. The spore concentrations and correspondingly released DPA fluorescence intensity were linearly correlated, and the LOD for the both Bacillus strains reached approximately 6800 spores $/ \mathrm{mL}$. The method developed in this study was verified to be efficient in the detection of endospores in Bacillus fermentation for probiotics production.

\begin{abstract}
Abbreviations
AU: arbitrary units; CFU: colony-forming units; CGMCC: China General Microbiological Culture Collection Center; CMCC: China National Center for Medical Culture Collections; CyDTA: cyclohexanediamine tetraacetic acid; DPA: dipicolinic acid; EDTA: ethylenediaminetetraacetic acid; EGTA: ethylene glycol tetraacetic acid; Eu: europium; HEDTA: 2-hydroxyethylethylenediamine triacetic acid; HPLC: high performance liquid chromatography; L-Ala: L-alanine: LB: Luria-Bertani; LOD: limit of detection; NSM: nutrient sporulation medium; OD: optical density; PMT: photomultiplier tube; Tb: terbium; UV: ultraviolet; SD: standard deviation.
\end{abstract}

\section{Authors' contributions}

Planning and designing of the study: XHG; Experimentation: XSL and CL; Data analysis: ZL; Manuscript drafting: XHG. All authors read and approved the final manuscript.

\section{Acknowledgements}

We would like to thank Xiaosheng Liang for his technical suggestions.

\section{Competing interests}

The authors declare that they have no competing interests.

\section{Availability of data and materials}

The data supporting the conclusions of this article are all included within the article.

\section{Consent for publication}

All authors gave their consent for publication.

Ethics approval and consent to participate

Not applicable.

\section{Funding}

This work was financially supported by the National Natural Science Foundation of China (No. 31672455), the Outstanding Young Talent Fund from Key Projects in Hubei Province Natural Science Foundation (NSF) (2018CFA077) and the Fundamental Research Funds for the Central Universities (CZT18002, CZT18003).

\section{Publisher's Note}

Springer Nature remains neutral with regard to jurisdictional claims in published maps and institutional affiliations.

Received: 11 May 2018 Accepted: 14 June 2018

Published online: 19 June 2018

\section{References}

Ai K, Zhang B, Lu L (2009) Europium-based fluorescence nanoparticle sensor for rapid and ultrasensitive detection of an anthrax biomarker. Angew Chem Int Ed Engl 48:304-308

Arnaud N, Georges I (2003) Comprehensive study of the luminescent properties and lifetimes of $\mathrm{Eu}^{3+}$ and $\mathrm{Tb}^{3+}$ chelated with various ligands in aqueous solutions: influence of the synergic agent, the surfactant and the energy level of the ligand triplet. Spectrochim Acta A 59:1829-1840

Bai Y, Wang Y, Goulian M, Driks A, Dmochowski IJ (2014) Bacterial spore detection and analysis using hyperpolarized 129 Xe chemical exchange saturation transfer (Hyper-CEST) NMR. Chem Sci 5:3197-3203

Chen ZM, Li Q, Liu HM, Yu N, Xie TJ, Yang MY, Shen P, Chen XD (2010) Greater enhancement of Bacillus subtilis spore yields in submerged cultures by optimization of medium composition through statistical experimental designs. Appl Microbiol Biotechnol 85:1353-1360

Cutting SM (2011) Bacillus probiotics. Food Microbiol 28:214-220

Fichtel J, Koster J, Rullkotter J, Sass H (2007) Spore dipicolinic acid contents used for estimating the number of endospores in sediments. FEMS Microbiol Ecol 61:522-532

Guo X, Li D, Lu W, Piao X, Chen X (2006) Screening of Bacillus strains as potential probiotics and subsequent confirmation of the in vivo effectiveness of Bacillus subtilis MA139 in pigs. Antonie Van Leeuwenhoek 90:139-146

Hazan R, Que YA, Maura D, Rahme LG (2012) A method for high throughput determination of viable bacteria cell counts in 96-well plates. BMC Microbiol 12:259

He J, Luo X, Chen S, Cao L, Sun M, Yu Z (2003) Determination of spore concentration in Bacillus thuringiensis through the analysis of dipicolinate by capillary zone electrophoresis. J Chromatogr A 994:207-212 
Hebert CG, Hart S, Leski TA, Terray A, Lu Q (2017) Label-free detection of Bacillus anthracis spore uptake in macrophage cells using analytical optical force measurements. Anal Chem 89:10296-10302

Heo J, Kim SK, Park KS, Jung HK, Kwon JG, Jang BI (2014) A double-blind, randomized, active drug comparative, parallel-group, multi-center clinical study to evaluate the safety and efficacy of probiotics (Bacillus licheniformis, Zhengchangsheng ${ }^{\circledR}$ capsule) in patients with diarrhea. Intest Res 12:236-244

Hindle AA, Hall EA (1999) Dipicolinic acid (DPA) assay revisited and appraised for spore detection. Analyst 124:1599-1604

Hong HA, Duc LH, Cutting SM (2005) The use of bacterial spore formers as probiotics. FEMS Microbiol Rev 29:813-835

Khardziani T, Kachlishvili E, Sokhadze K, Elisashvili V, Weeks R, Chikindas ML, Chistyakov V (2017) Elucidation of Bacillus subtilis KATMIRA 1933 potential for spore production in submerged fermentation of plant raw materials. Probiotics Antimicrob Proteins. https://doi.org/10.1007/s1260 2-017-9303-9

Pellegrino PM, Fell NF, Gillespie JB (2002) Enhanced spore detection using dipicolinate extraction techniques. Anal Chim Acta 455:167-177

Postollec F, Bonilla S, Baron F, Jan S, Gautier M, Mathot AG, Hallier-Soulier S, Pavan S, Sohier D (2010) A multiparametric PCR-based tool for fast detection and identification of spore-forming bacteria in food. Int J Food Microbiol 142:78-88
Pryor SW, Gibson DM, Hay AG, Gossett JM, Walker LP (2007) Optimization of spore and antifungal lipopeptide production during the solid-state fermentation of Bacillus subtilis. Appl Biochem Biotechnol 143:63-79

Rai NK, Rai AK (2008) LIBS-an efficient approach for the determination of $\mathrm{Cr}$ in industrial wastewater. J Hazard Mater 150:835-838

Reisfeld R, Saraidarov T, Panzer G, Levchenko V, Gaft M (2011) New optical material europium EDTA complex in polyvinyl pyrrolidone films with fluorescence enhanced by silver plasmons. Opt Mater 34:351-354

Ren H, Su YT, Guo XH (2018) Rapid optimization of spore production from Bacillus amyloliquefaciens in submerged cultures based on dipicolinic acid fluorimetry assay. AMB Express 8:21. https://doi.org/10.1186/s1356 8-018-0555-x

Rosen DL, Sharpless C, McGown LB (1997) Bacterial spore detection and determination by use of terbium dipicolinate photoluminescence. Anal Chem 69:1082-1085

Setlow P (2003) Spore germination. Curr Opin Microbiol 6:550-556

Vanderberg LA (2000) Detection of biological agents: looking for bugs in all the wrong places. Appl Spectrosc 54:376A-385A

Vasantha N, Freese E (1979) The role of manganese in growth and sporulation of Bacillus subtilis. J Gen Microbiol 112:329-336

Zhao S, Hu N, Huang J, Liang Y, Zhao B (2008) High-yield spore production from Bacillus licheniformis by solid state fermentation. Biotechnol Lett 30:295-297

\section{Submit your manuscript to a SpringerOpen ${ }^{\circ}$ journal and benefit from:}

- Convenient online submission

$\checkmark$ Rigorous peer review

- Open access: articles freely available online

- High visibility within the field

- Retaining the copyright to your article

Submit your next manuscript at $\boldsymbol{\nabla}$ springeropen.com 